\title{
MODELAGEM MATEMÁTICA DO PROCESSO DE EVAPORAÇÃO MULTI-EFEITO NA INDÚSTRIA DE PAPEL E CELULOSE
}

\author{
R. L. S. CANEVESI ${ }^{1}$, C. L. DIEL ${ }^{2}$, K. A. SANTOS $^{1}$, C. E. BORBA ${ }^{1}$, F. PALÚ ${ }^{1}$, E. A. DA SILVA ${ }^{1}$ \\ ${ }^{1}$ Universidade Estadual do Oeste do Paraná - Unioeste, Centro de Engenharia e Ciências Exatas. \\ ${ }^{2}$ Klabin Papéis Monte Alegre. Telêmaco Borba - PR. \\ E-mail para contato: rafael_canevesi@hotmail.com
}

\begin{abstract}
RESUMO - O processo de evaporação é amplamente empregado em diversos setores industriais, destacando-se o setor de papel e celulose. O emprego de modelos fenomenológicos e estatísticos consiste numa ferramenta utilizada na otimização de processos industriais. Os modelos fenomenológicos para este tipo de processo se baseiam em um conjunto de equações de balanço de massa, energia e de relações de equilíbrio, que devem ser resolvidas simultaneamente. Dessa forma, o presente trabalho tem como principal objetivo representar o processo de evaporação em regime permanente por meio de um modelo fenomenológico, empregando o método de Newton para sistemas não lineares e posteriormente empregar metodologias estatísticas para elaboração de um modelo empírico, avaliando a influência de variáveis como vazão, composição e temperatura de alimentação no consumo de vapor e na composição final. A vazão de alimentação de licor mostrou-se em ambos o caso como variável de maior influência, seguida pela composição de alimentação. Todavia, não foi possível encontrar um ponto ótimo no intervalo estudado.
\end{abstract}

\section{INTRODUÇÃO}

O processo de evaporação é empregado em diversões ramos da indústria, como a indústria de papel, açúcar e álcool, farmacêuticas, cloro, laticínios entre outros setores (Bhargava et al., 2008). Na indústria de papel e celulose normalmente emprega-se evaporadores do tipo casco tubo, empregando sistemas de evaporação em múltiplos efeitos (Empie, 2009).

Em uma planta de evaporação de múltiplos efeitos o vapor produzido pela primeira unidade evaporadora é utilizado em uma unidade posterior, tornando dessa forma o consumo de vapor menor que a utilização de um único efeito (Mccabe et al., 1993).

O setor de evaporação é responsável pelo consumo de uma parte significativa de energia demandada pela indústria. Dessa forma, qualquer alteração que vise aumentar a eficiência desse processo é de grande interesse industrial (Khanam e Mohanty, 2011). 
Assim, a utilização de modelos matemáticos para a obtenção de condições de operação que favoreçam a redução do consumo de energia consiste em uma alternativa largamente empregada (Bhargava et al., 2008).

Os modelos matemáticos empregados para esse processos consistem em sistemas de equações não lineares levando em conta as condições de alimentação, vapor e estrutura. Na literatura existem diversos modelos matemáticos para conjuntos de evaporação, como os propostos por Agarwal et al. (2004), Miranda e Simpsom (2005) e Bhargava et al. (2008).

Dessa forma, o presente trabalho tem como objetivo a elaboração de um modelo fenomenológico para o processo de evaporação multiefeito, posteriormente empregando análises estatísticas (delineamento composto central rotacional e análise de superfície de resposta) visando considerar a influência das variáveis do processo nas respostas do modelo.

\section{MODELAGEM MATEMÁTICA}

O modelo matemático foi desenvolvido para o sistema de evaporação apresentado na Figura 1, onde o fluxo de licor é contracorrente, existindo alimentação de vapor vivo no primeiro e segundo efeito. Os fluxo de licor e condensado são submetidos a expansão flash para a geração de uma quantidade de vapor adicional.

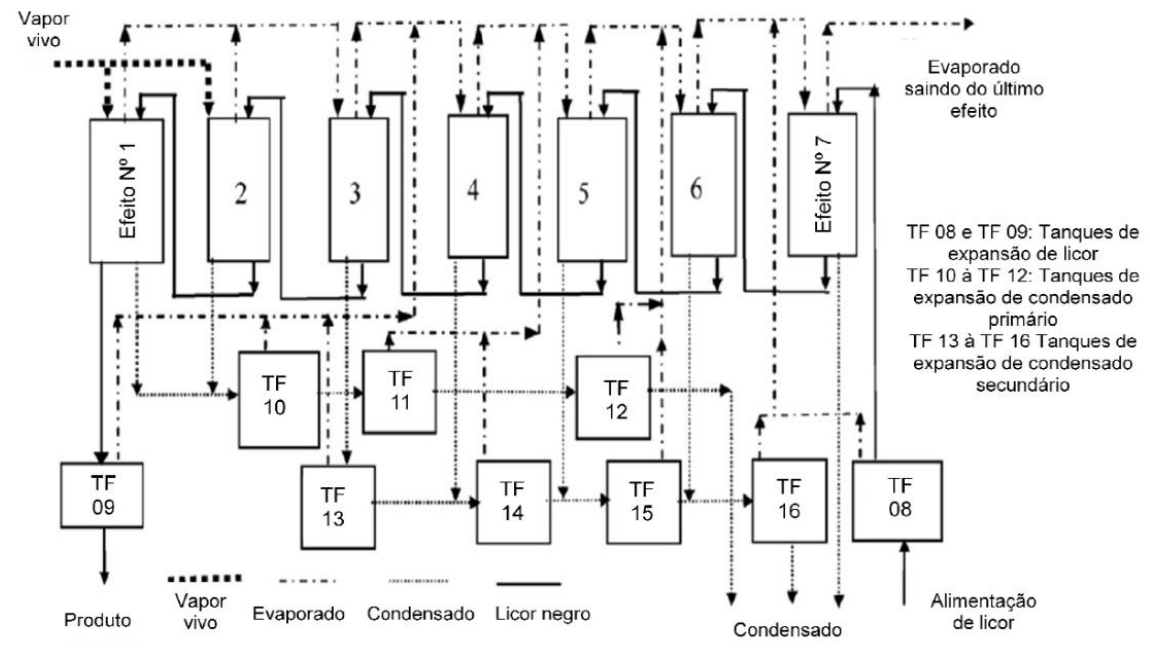

Fonte: Adaptado de Bhargava et al. (2008)

Figura 1 - Fluxograma do processo estudado por Bhargava et al. (2008).

O modelo matemático obtido tem como resposta todas as temperaturas e pressões de operação dos efeitos e unidades de flash, temperaturas, vazões, composições dos fluxos de licor, bem como todas as temperaturas e vazões dos fluxos de condenado e vapor. Para a resolução do sistema se faz necessário conhecer sete variáveis, que para neste caso consistem na temperatura do último evaporador, vazão, composição e temperatura de alimentação, razão entre as alimentações de vapor 
vivo, temperaturas do vapor vivo.

\subsection{Modelo Matemático Genérico para um Evaporador}

O balanço de massa global para o i-ésimo evaporador é representado matematicamente pela Equação (01)

$$
L E_{i}-L S_{i}-V S_{i}=0
$$

Já o balanço material para o componente sólido pode ser representado pela Equação (02).

$$
X E_{i} L E_{i}-X S_{i} L S_{i}=0
$$

O balanço de energia é representado pela Equação (03).

$$
L E_{i} h L E_{i}-L S_{i} h L S_{i}-V S_{i} H V S_{i}+Q^{t}=0
$$

Onde Q, consiste no calor recebido através da troca térmica, sendo representado matematicamente pela Equação (04).

$$
Q^{t}=A_{i} U_{i} \Delta T
$$

O balanço material para as correntes de vapor no evaporador é apresentado na Equação (05).

$$
V E_{i}-C S_{i}=0
$$

Já o balanço de energia para as correntes de vapor consiste na Equação (06).

$$
V E_{i} H V E_{i}-C S_{i} h C S_{i}-Q^{t}=0
$$
ideal.

Nas equações (3) e (6), as entalpias de vapor são calculadas admitindo comportamento de gás

\subsection{Modelo Matemático Genérico para uma Unidade Flash}

O balanço de massa global para a i-ésima unidade flash é semelhante a o aplicado aos evaporadores, representado pela Equação (01).

Nas unidades de flash de licor (TF08 e TF09) obtém-se também o balanço de massa para o componente solido, de forma análoga aos evaporadores pela Equação (2).

Já o balanço de energia para cada unidade flash é representado pela equação (07). 


$$
L E_{i} h L E_{i}-L S_{i} h L S_{i}-V S_{i} H V S_{i}=0
$$

Para as unidades flash de condensado (TF10-TF16) considera-se que o valor da composição $\left(X E_{i}\right.$ e $\left.X S_{i}\right)$ como igual a zero.

\subsection{Elevação do Ponto de Ebulição (EPE)}

Para o cálculo da elevação do ponto de ebulição emprega-se a relação funcional proposta por Ray et al. (1992), representada pela Equação (08).

$$
E P E=C_{1}\left(C_{2}-X\right)
$$

Onde $X$ consiste na fração de sólidos $C_{1}$ e $C_{2}$, parâmetros retirados de Bhargava et al. (2008).

\subsection{Capacidade Calorifica do Licor (Cp)}

Para o cálculo das entalpias dos fluxos de licor faz-se necessário o conhecimento do valor da capacidade calorífica do mesmo, todavia, ele apresenta dependência funcional com relação ao teor de sólidos do licor. Bhargava et al. (2008) emprega uma correlação, representada matematicamente pela Equação (09).

$$
C p_{L}=4,187(1-0,54 x)
$$

Onde $x$ consiste na fração de sólidos em dispersão na corrente.

\subsection{Coeficiente Global de Troca Térmica (U)}

O coeficiente global de troca térmica $\left(\mathrm{kJm}^{2} \mathrm{~K}-1\right)$ consiste em uma função de diversos fatores, que, dentre os mais importantes, pode-se citar três: composição, gradiente de temperatura e vazão. Dessa forma, Bhargava et al. (2008) propôs um correlação empírica para o cálculo do coeficiente U, representada matematicamente pela Equação (10).

$U=2\left(0,6\left(\frac{\Delta T}{40}\right)^{-, 37}\left(\frac{x}{0,6}\right)^{-1,23}\left(\frac{F}{25}\right)^{0,07}\right)$

Os valores da composição e vazão empregados na Equação (10) consistem na média aritmética entre a entrada e saída de licor do evaporador.

\subsection{Pressão de Vapor}

Para o cálculo da pressão de operação dos evaporadores, bem como unidades flash emprega-se a equação de Antoine, utilizando-se parâmetros retirados de Smith et al. (2007). 


\subsection{Resolução do Sistema não Linear}

O Método de Newton consiste no método mais amplamente estudado e conhecido para resolução de sistemas de equação não lineares. No caso de uma equação não linear a uma variável, o método de Newton consiste em se tomar um modelo local linear da função $f(x)$ em torno de $x_{k}$, e este modelo é a reta tangente à função em $x_{k}$.

Para um sistema de equações não lineares, tem-se conhecida a aproximação dada pela equação 11.

$f_{i}(x)=f_{i}\left(x_{k}\right)+\nabla f_{i}\left(x_{k}\right)^{T}\left(x-x_{k}\right)$

E portanto, sendo $\mathrm{F}(\mathrm{x})$ o vetor de funções que compõe o sistema de equações, resultando na seguinte equação matricial:

$$
F(x)=F\left(x_{k}\right)+J\left(x_{k}\right)\left(x-x_{k}\right)
$$

Considerando que $\left(x-x_{k}\right)$ como sendo $S_{k}$ obtém-se a seguinte equação:

$J\left(x_{k}\right) S_{k}=-F\left(x_{k}\right)$

Onde $J(x)$ consiste na matriz jacobiana e $F(x)$ o vetor com as funções que compõem o sistema de equações não lineares, ambas avaliadas em $x_{k}$, e $s_{k}$ consistem no incremento para cada variável para a próxima interação. Para a utilização do método se faz necessária à fixação de duas tolerâncias, a primeira para satisfazer as condições presentes no vetor $F(x)$, e a segunda para o tamanho mínimo do passo $s_{k}$.

\subsection{Análise Estatística}

Submeteu-se o modelo a metodologia de delineamento composto central rotacional (DCCR), com o intuito de identificar a influência das variáveis referente a corrente de alimentação de licor no sistema de evaporação multi-efeito, bem como a obtenção de um modelo estatístico. Os fatores, bem como os níveis estudados são apresentados na Tabela 2.

\begin{tabular}{ccccc}
\hline Fator & $\mathbf{( - 1 , 6 8 )}$ & $\mathbf{( - 1 )}$ & $\mathbf{( + 1 )}$ & $\mathbf{( + 1 , 6 8 )}$ \\
\hline Alimentação de Licor $\left(\mathrm{kg} \mathrm{s}^{-1}\right)$ & 11.67 & 13.27 & 17.95 & 19.55 \\
Composição de Alimentação $(\%)$ & 9.82 & 10.62 & 12.98 & 13.78 \\
Temperatura de Alimentação $\left({ }^{\circ} \mathrm{C}\right)$ & 59.3 & 61.5 & 67.9 & 70.1 \\
\hline
\end{tabular}

\section{RESULTADO E DISCUSSÃO}

O modelo matemático obtido foi testado com as variáveis utilizadas como entrada por Bhargava et al. (2008), comparando os resultados obtidos com os apresentados pelo autor. Pode-se visualizar na Figura 2 que os resultados obtidos diferem em algumas variáveis em virtude de considerações diferenciadas com relação ao equilíbrio de cada evaporador. Também se observa que a composição de saída apresenta pouca diferença entre os resultados obtidos por ambos os trabalhos. 


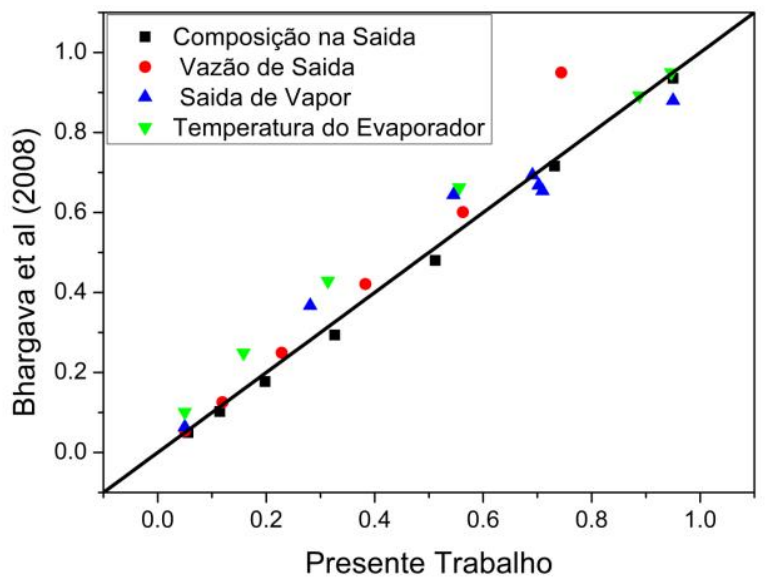

Figura 2 - Gráfico de comparação entre os resultados normalizados obtidos pelo presente trabalho e por Bhargava et al. (2008).

O planejamento experimental DCCR foi empregado para avaliar a influência dos fatores nas variáveis: Composição de Saída e Consumo de Vapor, sendo os resultados obtidos apresentado na figura 3 na forma de gráficos de Pareto.

(a)
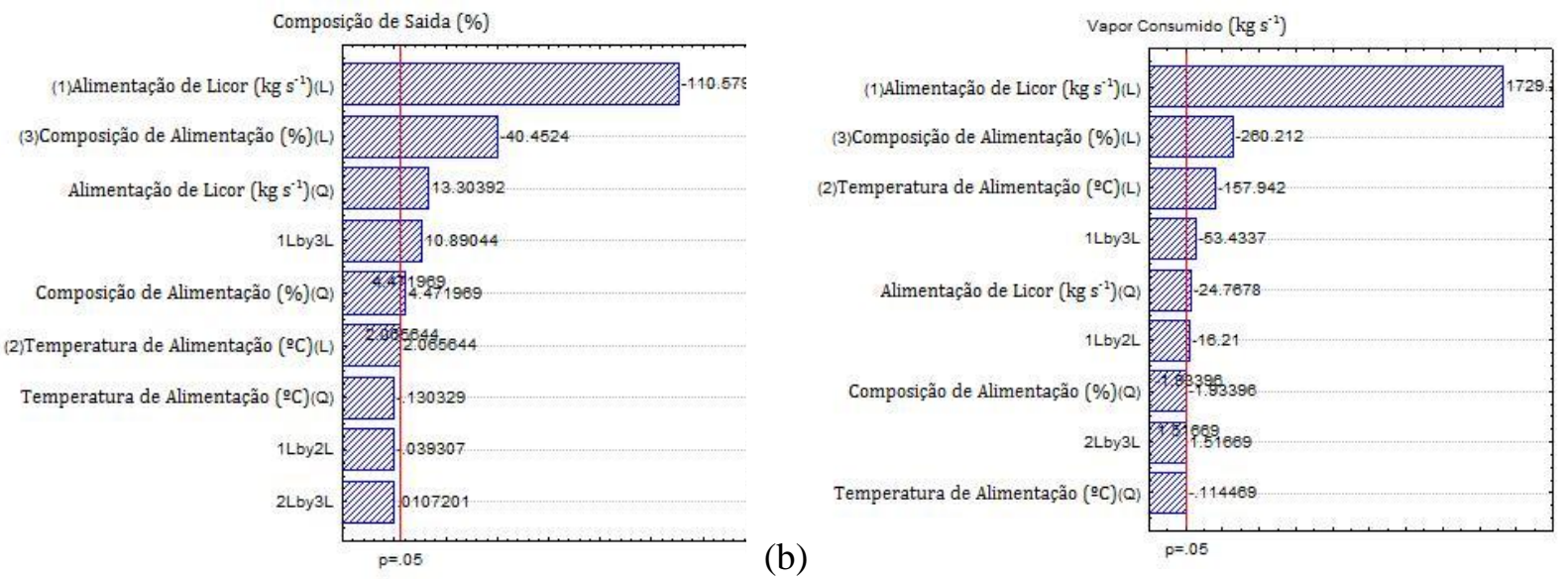

Figura 3 - Gráfico de Pareto: (a) para a Composição de Saída; (b) para Vapor Consumido.

Observa-se na Figura 3 que para a composição de saída o fator que apresenta maior significância consiste na Vazão de Alimentação. Também mostrou significância estatística a composição de alimentação e a sua interação com a vazão de alimentação. $O$ fator temperatura da alimentação não mostrou influência na composição de saída para o nível de significância de $5 \%$. Desta forma, representa-se o comportamento da composição final do licor a partir da superfície de resposta apresentada na Figura 4, em função dos dois fatores que se mostraram significantes. 


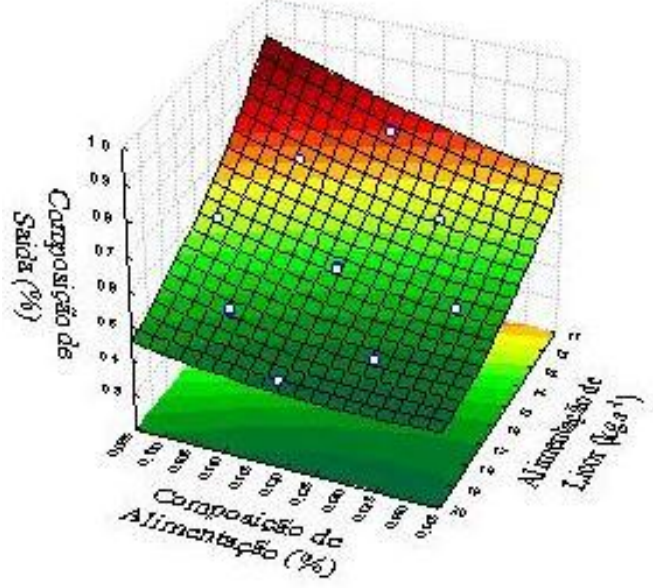

Figura 4 - Superfície de resposta para a variável composição de Saída em função da composição e Vazão de alimentação.

Por meio da Figura 4 nota-se que a composição de saída sofre significativa influência positiva com a redução da vazão de alimentação de licor, enquanto que a redução da porcentagem de sólidos na alimentação apresenta influência inferior no aumento da composição de saída.

Observando a Figura 3 (b) nota-se que para a variável Consumo de Saída todos os fatores se mostraram significativos na forma linear, e somente a vazão de alimentação se mostrou significativo na forma quadrática. Observa-se também que entre as interações somente a composição entre a Temperatura de Alimentação e a composição de alimentação não se mostraram influentes no nível de significância estudado.

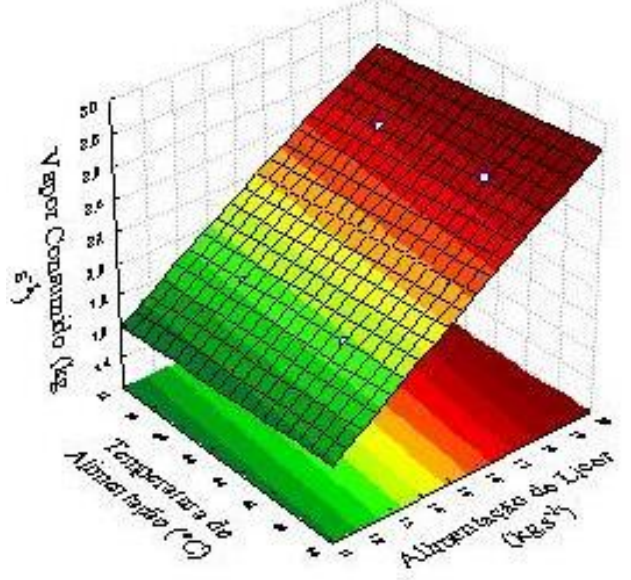

(a)

Figura 5 - Superfície de resposta para a variável Consumo de Vapor em função: (a) Temperatura e Vazão de Alimentação; Composição e Vazão de Alimentação. 
A partir da Figura 5 (a) e (b) nota-se que a Vazão de alimentação influência fortemente no consumo de vapor do processo, enquanto que a Temperatura de Alimentação e Composição de Alimentação influenciam com menor intensidade na mesma variável.

\section{CONCLUSÃO}

O presente trabalho conseguiu reproduzir com boa eficiência os resultados obtidos por Bhargava et al. (2008). A Metodologia de planejamento experimental DCCR mostrou que entre os fatores analisados somente a vazão e a composição de alimentação influenciam na composição de saída. Já para a o consumo de vapor todos os fatores, ao menos em sua forma linear se mostraram significativos. Todavia, não foi possível a obtenção de um ponto ótimo para o processo em função do intervalo estudado não apresentar pontos máximos ou mínimos, como mostrado nas superfícies de respostas.

\section{AGRADECIMENTOS}

Ao CNPq e a Klabin Papéis Monte Alegre pelo financiamento do projeto.

\section{REFERENCIAS BIBLIOGRÁFICAS}

AGARWAL, V. K., ALAM, M. S.,; GUPTA, S. C.; Mathematical model for existing multiple effect evaporator systems. Chem. Eng. World, v.39, p.76-78, 2004.

BHARGAVA, R.; KHANAM, S.; MOHANTY, B.; RAY, A. K., Simulation of flat falling film evaporator system for concentration of black liquor, Comput. Chem. Eng., v.32, p. 3213-3223, 2008.

EMPIE, H. J.; Fundamentals of the "kraft" Recovery Process, Atlanta: Tappi Press, 2009.

KHANAM, S.; MOHANTY, B.; Development of a new model for multiple effect evaporator system, Comput. Chem. Eng.,v.35, p.1983-1993, 2011.

MCCABE, W.L., SMITH, J.C., HARRIOT, P.; Unit Operations of Chemical Engineering, ed. New York, McGraw-Hill, 1993.

MIRANDA, V. e SIMPSON, R.; Modelling and simulation of an industrial multiple effect evaporator: Tomato concentrate, J. Food Eng., v.66, p.203-210, 2005.

RAY, A. K.; RAO, N. J.; BANSAL, M. C.; MOHANTY, B.; Design data and correlations of waste liquor/black liquor from pulp mills. IPPTA Journal, v. 4, p.1-21, 1992.

SMITH, J. M.; VAN NESS, H. C.; ABBOTT, M. M.; Introducao a Termodinamica da Engenharia Química, LTC, 2007. 